\title{
Graphene Nanoribbon Simulator of Vacancy Defects on Electronic Structure
}

\author{
Kien Liong Wong, Mohamad Azri Sufi Mahadzir, Wee Khang Chong, \\ Mohd Shahrizal Rusli, Cheng Siong Lim, Michael Loong Peng Tan \\ School Electrical Engineering, Faculty of Engineering, Universiti Teknologi Malaysia \\ UTM Skudai Johor, 81310, Malaysia
}

\section{Article Info \\ Article history: \\ Received Jul 11, 2018 \\ Revised Sep 6, 2018 \\ Keyword: \\ Band structure \\ Density of states \\ GNR \\ MATLAB \\ Vacancy}

Accepted Sep 20, 2018

\begin{abstract}
Graphene Nanoribbon Simulator (GNRSIM) is developed using MATLAB Graphical User Interface Development Environment to study the electronics properties of graphene nanoribbons (GNRs). The main focus of this research is the simulation effects of single vacancy 1 in graphene nanoribbons lattices on electronic structure. The band structure and density of states are explored by using tight binding approximation where a Hamiltonian operator with nearest-neighbor interactions is introduced. The simulator has a wide range of input parameters where user can select armchair or zigzag GNR. The size of the lattices namely width and length can be varied. The location of the vacancy defect can be pinpoint by providing the row and column of the missing atom. The limitation of GNRSIM at present is that it can only accept a single atom vacancy. GNRSIM is able to be executed as a standalone application software in understanding the fundamental properties of semiconductor material and device engineering through ab-initio calculations.
\end{abstract}

Copyright $@ 2018$ Institute of Advanced Engineering and Science. All rights reserved.

Corresponding Author:

Michael Loong Peng Tan,

School Electrical Engineering, Faculty of Engineering, Universiti Teknologi Malaysia,

UTM Skudai Johor, 81310, Malaysia.

Emailmichael@utm.my

\section{INTRODUCTION}

Technological advances and innovations are key catalysts towards new discoveries on new materials that will revolutionize electronic devices. Worldwide, people have had to deal with electronic gadgets, leading to many advantages and disadvantages. As the use of electronic gadgets has become a part of human daily life in this modernization, with a multitude of uses at consumers' fingertips, research must explore this area further. Gadgets have become a medium for communication, entertainment, computation and other types of applications. Electronic gadgets, or electronic devices, are commonly composed of metal-oxide semiconductors (MOS) in integrated circuits (ICs). In our industry, Moore's Law must be understood. This rule has shifted its driving-forces more than once before; from counting transistors that the industry pivoted, to transistor size-and cost-scaling due to the limits of on-chip size and complexity [1].

Figure 1 shows the timeline of the number of transistor count in a chip since 1971 [2]. This figure shows the plotting of the CPU with the numbers of transistors versus the dates of introduction. The point on the graph has followed the logarithmic vertical scale and the line is corresponding to exponential growth with number of transistor doubling every two years. Graphene, a one-of-a-kind 2-Dimensional material, one atom thin substance with superior electrical properties that has contributed largely to the progression of postCMOS technology.

In 2004, a single layer of carbon atom was found that would spur the electronic innovation. Two physicists, Andre and Konstantin from University of Manchester, concocted this leap forward and won the 
Nobel Prize in 2010 [3, 4]. By utilizing a simple scotch tape method, graphene was extracted from graphite from repeated sticking and peeling. This exceptional two-dimensional material was pivotal to grapheneCMOS technology.

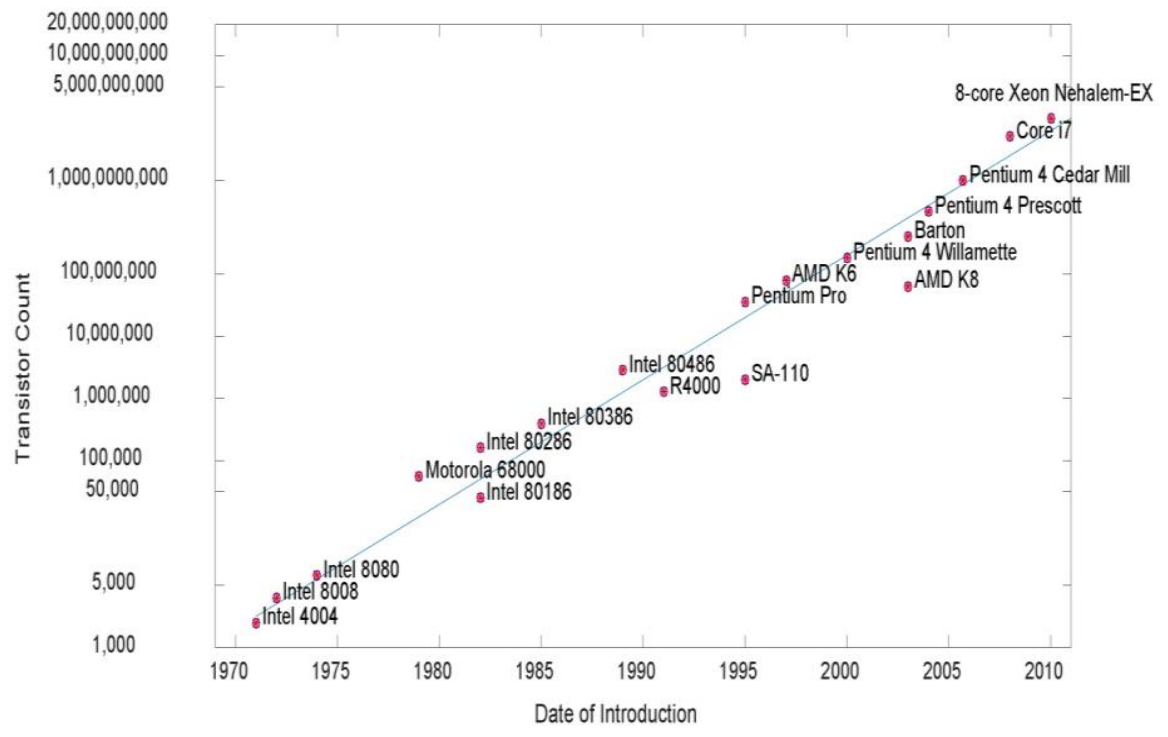

Figure 1. Microprocessor transistor counts 1971-2011 with moore’s law

In this research analysis, an educational simulation tool is presented to enhance understanding of the fundamentals of graphene's structural and electrical properties. User can explore and observe the electrical properties of GNR where there is a missing atom or atom vacancy in the lattice structure. Graphene nanoribbon simulator or also known as GNRSIM is developed using MATLAB graphical user interface (GUI) software. At present, there are various simulation tools that can be used as educational kits in the learning and teaching process. Unfortunately, most simulation tools are quite expensive because they require a license. In some cases, a simulation tool that has a great interface and navigation on graphene properties can only be used for experiments and then ask users to get their licenses. The fall of the majority of these simulators is that they only focus on one graphene application. In the end, we will have a variety of simulation tools that uses different formalism that do not provide consistent results for a specific structure. Additionally, existing simulation tools may be too complex for researcher to navigate as they are accompanied by unknown parameters that are taken into account when used. The simulation tool developed in this research can extract data and simulate it according to the selected properties. The most focused features of the graphene are their bandgap. Therefore, this research will be subject to structure of atomic structure, chirality index and wide calculators. By simply selecting properties and by inputting multiple inputs in advanced GUI simulators, users can explore different electrical properties on the device.

\subsection{Graphene}

Graphene a one-atom-thick layer of carbon atoms orchestrated in a hexagonal cross section [4, 5]. Graphene is the building-piece of graphite that is utilized in pencil lead, yet graphene is a momentous substance by itself - with a variety of amazing properties. It also has high transparency, is flexible, and has low resistivity which is about $10^{-6} \mathrm{ohm} . \mathrm{cm}$. Graphene is the slenderest material known to man at one atom thick, and furthermore extraordinarily solid - around 200 times more grounded than steel [3]. There are ongoing research to learn the remarkable properties of graphene and identify potential applications in nanoelectronics, optoelectronics, biosensor, solar cells, and water filters, among many others.

The structure shown in Figure 2 is must be highlighted due to the note that it is through these bonds, that the electronic properties of graphene appear, allowing free moving electrons which inhibits its special characteristics [6]. This concept was utilized in the simulation of band structure of a single graphite layer using tight binding approximation. The electronic properties are affected by the bonding of $\mathrm{sp}^{2}$ hybrids of each carbon atoms in graphene. The carbon forms three covalent $\sigma$ bonds at an angle of $120^{\circ}$. Additionally, a high electrical conductivity is achieved when $\pi$ electron is able to move freely between carbon atoms [7]. 


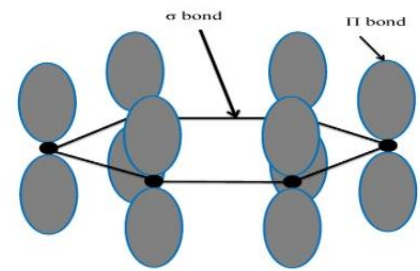

Figure 2. Bond in graphene

\subsection{Existing Graphene FET Models}

Our ongoing research on graphene FET has led to the development of this simulation tools in vacancy defect. In our previous work [8], a tight-binding model of GNR is created by collapsing quasi-2D structure of GNR into equivalent 1D matrix system via alpha and beta matrices, which describes the interactions in the unit cell and between unit cells respectively. The tight-binding model is sufficient and accurate enough for hydrogen-passivated-edge GNR, and Hartree's assumptions are valid due to the small size of carbon atoms. In this work, the vacancy is introduced in the defective Hamiltonian matrices by modifying the alpha and beta matrices. Note that users must have a firm understanding of these configurations to define the desired defective Hamiltonian operator for simulation.

\subsection{Graphene Nanoribbon Structure}

GNR can be categorized into two different structures with different electronic properties [8]. The two structures of the GNR are armchair-edge graphene nanoribbon (AGNR) and zigzag-edge graphene nanoribbon (ZGNR) [9]. The structure has described their edge type of GNR which can be determined by looking at the edge of the channel orientation. Thus, the horizontal channel length, the orientation of channel and the edges of the shape will determine whether the structure is armchair or zigzag. Figure 3 below has shown both structures of the graphene [10].

$$
\mid
$$

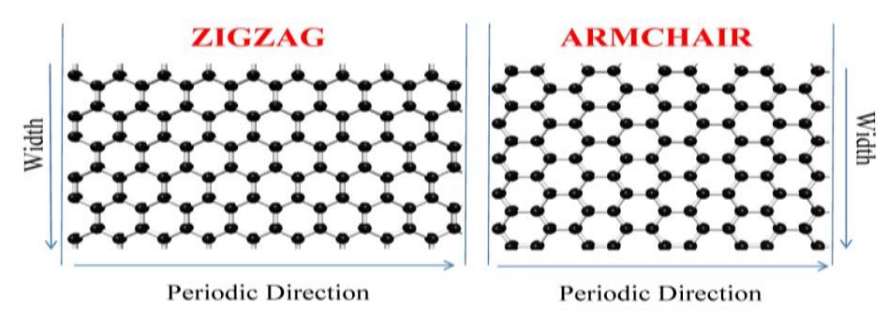

Figure 3. Structure of AGNR and ZGNR

\subsection{Band Structure GNRFET}

Band structure speaks to the conduction band and valence band as seen in Figure 4, and there are numerous electronic properties that can be separated by the different states of band structure [11]. On the off chance that the conduction and valence band meet at the K-purpose of Brillouin zone, the structure is gapless as depicted in Figure 4(i) and has electronic properties of metal. When the conduction and valence bands are isolated at a similar point, this would force semiconducting electronic properties as shown in Figure 4 (ii). In this condition, graphene can be switched on and off and therefore can be used for logic applications [12].

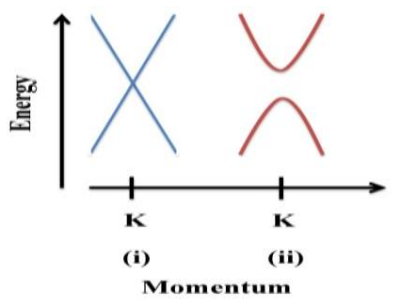

Figure 4. Band structure around K-point of (i) Large area graphene, (ii) Graphene nanoribbons 


\subsection{Density of States}

The DOS infers to the quantity of density states at a given density level, $\mathrm{E}$ towards $\mathrm{E}_{\mathrm{c}}$ or $\mathrm{E}_{\mathrm{v}}$. Inhabitance of the states at every energy can be utilized to calculating the quantum transport in semiconductors. The DOS in a semiconductor rises to the density per unit volume and depicts the number density of allowed quantum states. Figure 5 is the sample of an expected result for density of states plot which would vary according to the width of the graphene sheets [13]. The DOS of a specified device will always exist in two energy band, that are called the conduction band and the valence band. It is very important for researchers or engineers to understand the characteristics of DOS as it would provide the information on its electrical work input and output per electron [10].

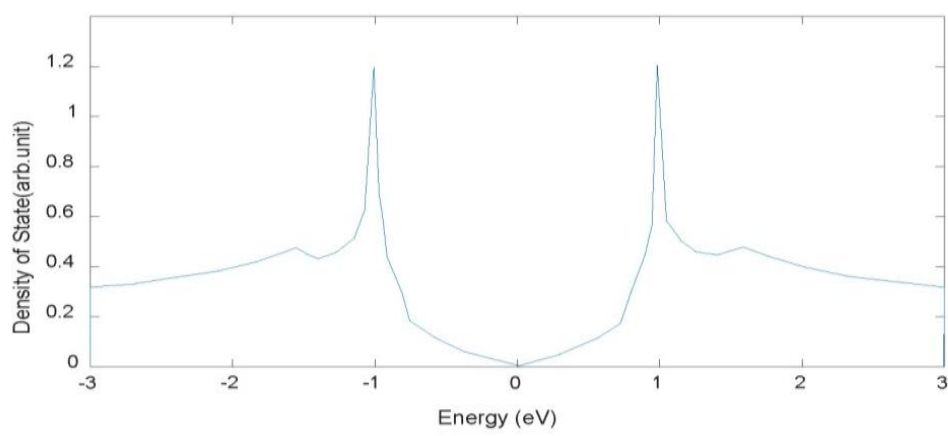

Figure 5. Density of states for idealized graphene

Figure 6 demonstrates the electronic bandstructure, density of states functions for 3-dimensional structure $\mathrm{g}(\mathrm{E})$, Fermi-Dirac probability function $\mathrm{f}(\mathrm{E})$, and areas representing electron and hole concentrations for the case when Fermi energy, $\mathrm{E}_{\mathrm{F}}$ is near the midgap energy. The carrier concentration essentially the product of $g(E)$ and $f(E)$. As such, the insight into band structures and DOS are important into the physics of semiconductor.

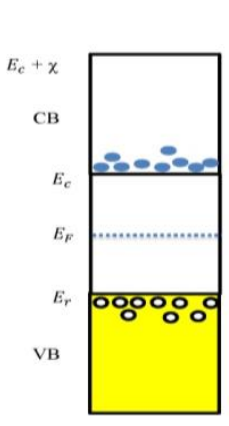

(a)

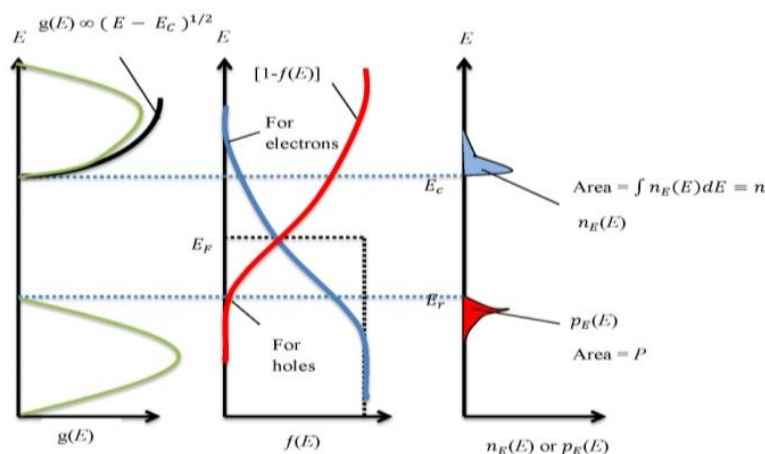

(c)

(d)

Figure 6. Calculation of electron or holes concentration for a 3-dimensional structure (a) Energy band structure (b) Density of states DOS (c) Fermi-dirac probability function (d) The product of $g(E)$ and $f(E)$ is the density of electrons in conduction band. area indicates the concentration

\subsection{Vacancy of Graphene Atom}

Atomic vacancy has a strong impact on the mechanical and electronic properties of graphene. We have demonstrated how a single opening modify the density of states and band structure of GNR through tight-binding modeling. Figure 7 depicts the occurrence of a single vacancy defect when one carbon atom is missing in GNR, resulting in that a total of three bonds is lost which are between missing atom and atom $\mathrm{A}, \mathrm{B}$ and $\mathrm{C}$, respectively. 


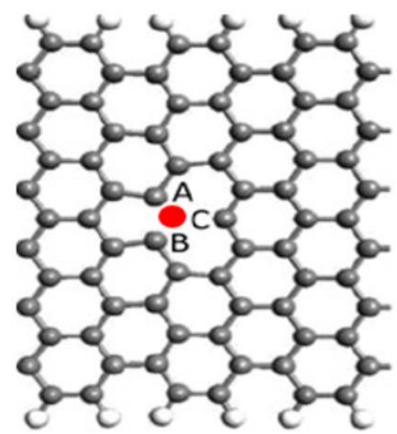

Figure 7. Single vacancy defect and lost of bonds

\section{RESEARCH METHOD}

The main target in developing the simulation tool is to allow the user to observe the atomic structure, width plot and missing atoms of graphene. The development of the simulation tool is based on the previous modelling work [8], where an ideal GNR model is introduced using the numeric computational approach on MATLAB. Schrödinger's equation is time - independent as written in general form:

$$
E\{\Psi\}=[H]\{\Psi\}
$$

As in the eigenvalue equation, the wave function in (1) is said to be eigenvalue of Hamiltonian operator. It contains the detailed information of the physical system including the position of constituent particles and the interaction among each other [14]. Once the Hamiltonian being paired up with respective wave function, the available energy spectrum that exist for the particular system can be obtained [15]. The defect properties could be well exhibited in the Hamiltonian operator. The structural defect applied on GNR causes the changes in the interactions among the carbon atoms in GNR, which directly affect the components in that particular Hamiltonian operator.

The tight binding model is solved using Non-Equilibrium Green's Function (NEGF) formalism [1618]. Datta recommends three simplifying assumptions for device modeling [19]. Firstly, the utilize of basis function adapted from Hartree's assumption [20], which states that the number of single electron wave function equals to the number of electrons in the respective system, that permits one to define the system by a single-particle electron method. Secondly, these wave functions are only affected by their nearest neighbours. Finally, the discretization of the Hamiltonian operator and its basis functions into matrix equations. For a one dimensional system with three hydrogen atoms and $1 \mathrm{~s}$ orbital wave function, the Hamiltonian matrix of the three hydrogen atoms can be constructed as (2), where $u_{11}, u_{22}$ and $u_{33}$ represent the $1 \mathrm{~s}$ orbital wave function. The term $u_{11}, u_{22}$ and $u_{33}$ are the self-interacting energies, while $u_{12}, u_{21}, u_{23}$ and $u_{32}$ are the interacting energies between respective nearest neighbour atoms [8]. The single vacancy defect inside the atomic structure of GNR can be introduced inside the Hamiltonian matrix by removing the interacting energy at the defect location.

$$
E\left\{\begin{array}{c}
\Psi_{1} \\
\Psi_{2} \\
\Psi_{3}
\end{array}\right\}=\left[\begin{array}{lll}
u_{11} & u_{12} & 0 \\
u_{21} & u_{22} & u_{23} \\
0 & u_{32} & u_{33}
\end{array}\right]\left\{\begin{array}{c}
\Psi_{1} \\
\Psi_{2} \\
\Psi_{3}
\end{array}\right\}
$$

The disintegration of a quasi-two dimensional GNR structure into a one-dimensional structure is done by collapsing in the width direction into a single matrix form. They are defined as the alpha matrix, $\alpha$, while the beta matrix, $\beta$ is defined to depict the interaction between the alpha matrixes. The number of rows in alpha and beta matrices equals to the number of carbon atoms present in a GNR unit cell. Throughout the research, the numbering sequence of these $\alpha$ and $\beta$ during calculation are strictly followed. Once both the alpha matrices and beta matrices are defined, the results are aggregate to produce the final Hamiltonian matrix. For example, the Hamiltonian matrix for $4-$ ZGNR/AGNR with length of 3 is shown in Equation 3. 


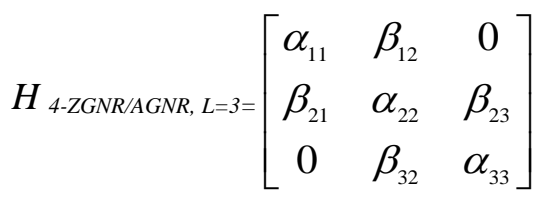

With the firm concepts of tight binding model, the design interface is generated using the MATLAB GUI. After that, the MATLAB algorithm is coded so that the layout of GUI is functioning accordingly so that the interface can be designed to improve the operation of the simulator tool.

\subsection{GNRSIM User Manual}

In navigating through the simulator, the user needs to follow the steps shown in Figure 9. For the purpose of fundamentally understanding graphene nanoribbons, the electronic properties included in GNRSIM are the band structure, DOS, and device specifications. There are three steps that the user needs to follow in using this simulator: one, the user must load the data input into the six parameters correctly for the type, width, length, row defect, column defect, and defect column position. The user needs to key in appropriate values at the input parameters panels. To select the type of edges of graphene nanoribbon, the user has to key in value one for an AGNR and value two for ZGNR. Other values than those specified will cause an error and there will be no output display. Next, the important parameter that requires user attention is the parameter of width. Bear in mind that the structure of AGNR requires the minimum widths of 3 to show as an AGNR structure, while for ZGNR the minimum widths is 2. Also, the important parameter needed to consider on defect column position is that character " $A$ " will be for the left side of graphene, while character "B" will be for the right side of graphene. For the second step, the user can choose any desired property to be simulated on the interface like the figure of atomic structure of graphene, the four-figure density of the state of graphene, and the band structure of the graphene. The simulation result will be shown on the graphic display on the left side of the simulator. Navigation tool cursor data can be used to plot the exact value of the graph shown on the axes. Lastly, the user can use the reset button to reset all the parameters loaded into zeros value and the graphical result will also be reset.

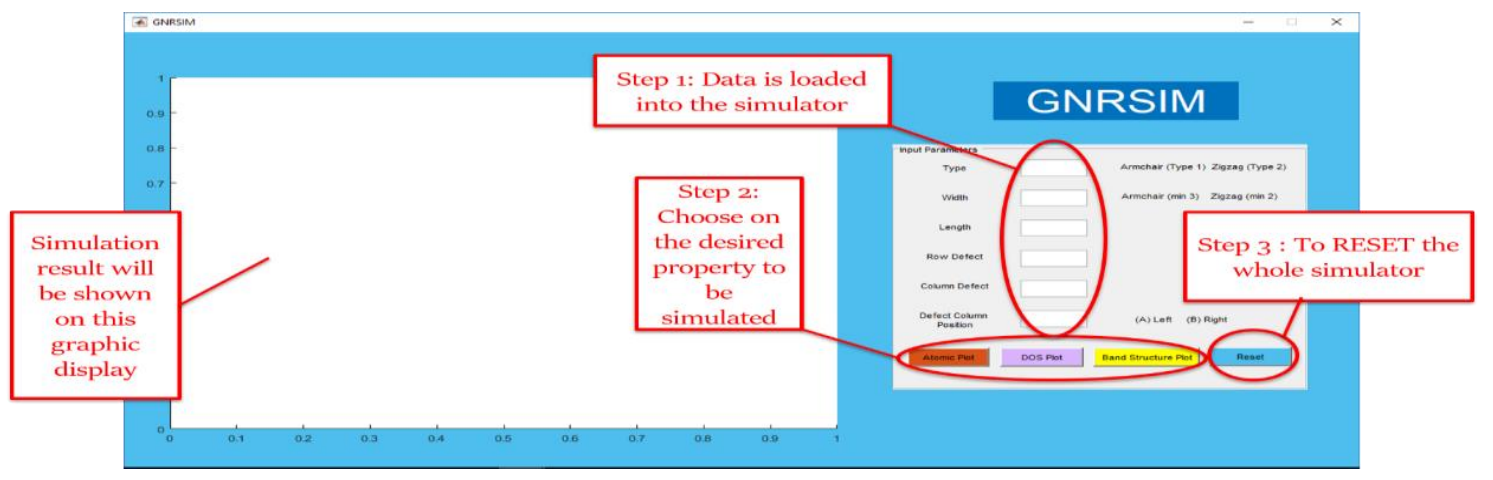

Figure 9. Instruction of GNRSIM

\section{RESULTS AND ANALYSIS}

The interface of the simulator tool, GNRSIM, is generated by using MATLAB GUIDE, as the application is embedded with the MATLAB software. GNRSIM is expected to be able to deliver educationally, and is equipped with an easy-to-use graphical user interface including a push button, editable input parameters and a graph plotter for information analysis. Predefined tools can be added to the simulator such as pan, zoom in and zoom out, data cursor and rotate that allows user to navigate around the axes panel.

\subsection{GNRSIM: Atomic Plot}

The atomic plot is represented in Figure 10 showing 4-AGNR. The input parameter is stated in Table 1. This simply means that the structure's widths equal 4. With the same inputs, the device specifications of bandgap, device width, device length, device row defect, device column defect, device defect column position and electrical property were extracted. Figure 11 shows the atomic plot of 4-ZGNR and the input parameters are stated in Table 2. From this analysis, the user should be able to conclude that 
graphene electrical properties are affected by the edge type. Armchair inhibits the possible electrical property of being semiconducting or metallic and the zigzag edges are always metallic which also means that there is no bandgap.

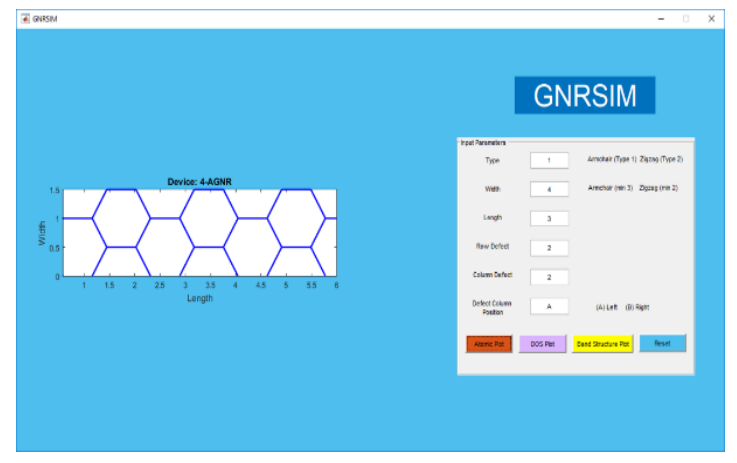

Figure 10. Atomic structure of 4-AGNR

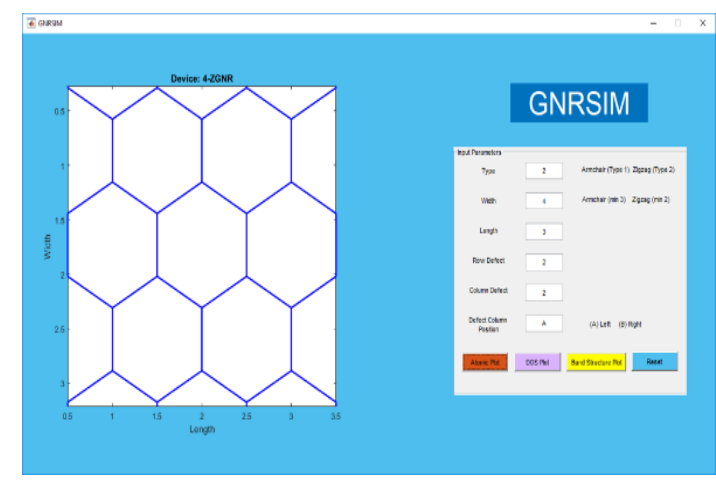

Figure 11. Atomic structure of 4-ZGNR
Table 1. Input Parameters of 4-AGNR for Atomic Structure of Graphene

\begin{tabular}{lc}
\hline \multicolumn{1}{c}{ Input Parameters } & Value \\
\hline Type & 1 \\
Width & 4 \\
Length & 3 \\
Row Defect & 2 \\
Column Defect & 2 \\
Column Defect Position & $\mathrm{A}$ \\
\hline
\end{tabular}

Table 2. Input Parameters of 4-ZGNR for Atomic Structure of Graphene

\begin{tabular}{lc}
\hline \multicolumn{1}{c}{ Input Parameters } & Value \\
\hline Type & 2 \\
Width & 4 \\
Length & 3 \\
Row Defect & 2 \\
Column Defect & 2 \\
Column Defect Position & $\mathrm{A}$ \\
\hline
\end{tabular}

\subsection{GNRSIM: Band Structure}

The band structure is plotted when the user presses the function button band structure after keying in the input parameters. The user will be able to identify that different AGNR and ZGNR have their own shape of band structure. AGNR enables control of the on/off switching ratio of a device application as the band gap can be fabricated according to the width. It is revealed that specific widths of the device will impose specific electrical properties. There are two types of electrical properties: semiconducting have the gap between the valence band and conduction band, and metallic has no energy gap. The property of band structure is selected, and the user will have to key in all the necessary inputs before pressing the Band Structure button. Figure 12 proves that the results in constructing the band structure were validated and the inputs to gain the structure are as shown in Table 3. Figure 13 described the band structure of ZGNR with chirality of 4 unit cell (UC) width. The user will be able to observe the major differences in that each type of edge has their own shape of band structure which shows their electronic characteristics. Table 4 is the input parameters to plot the band structure. 


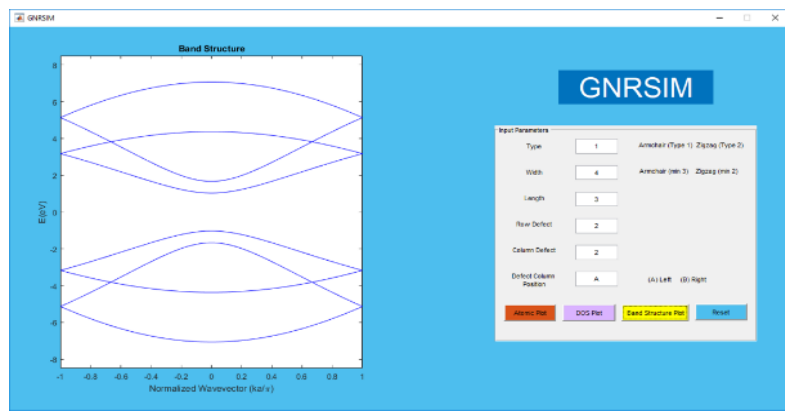

Figure 12. Band structure of 4-AGNR

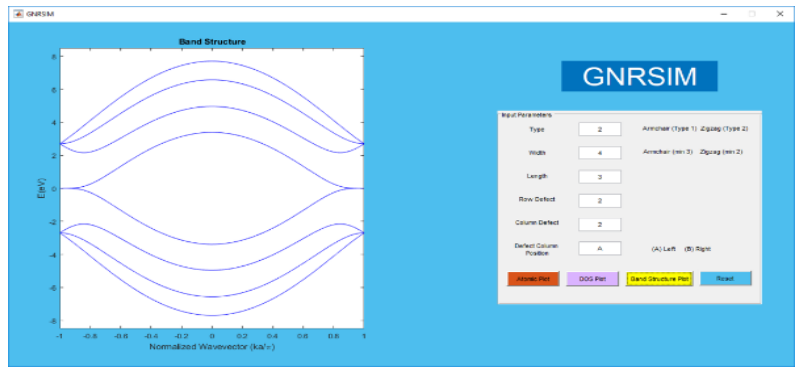

\begin{tabular}{lc}
\begin{tabular}{c} 
Table 4. Input Parameters of \\
\multicolumn{2}{c}{ Band Structure of Graphene }
\end{tabular} \\
\hline \multicolumn{1}{c}{ Input Parameters } & Value \\
\hline Type & 2 \\
Width & 4 \\
Length & 3 \\
Row Defect & 2 \\
Column Defect & 2 \\
Column Defect & \\
Position & A
\end{tabular}

Figure 13. Band structure of 4-ZGNR

\subsection{Density of States}

Density of states is an extension of band structure as it will find the states of each energy level being represented by each sub-band which can also be referred to as the wave vector. This provides insight for users and researchers to find out which energy level will have the highest density of electrons. As the user presses the button to simulate density of states, GNRSIM will load the input data and compute the Eigen energy based on the sub-bands energy level and plot them. Figure 14 and Table 5 include the data used to plot the graph of density of states. To increase the density of states for carrier, the length of the structure is increased from 3 to 10 unit cell (UC). The major drawback of increasing the length is that this will consume high computational time during simulation. The results are shown in Figure 15 and Table 6.

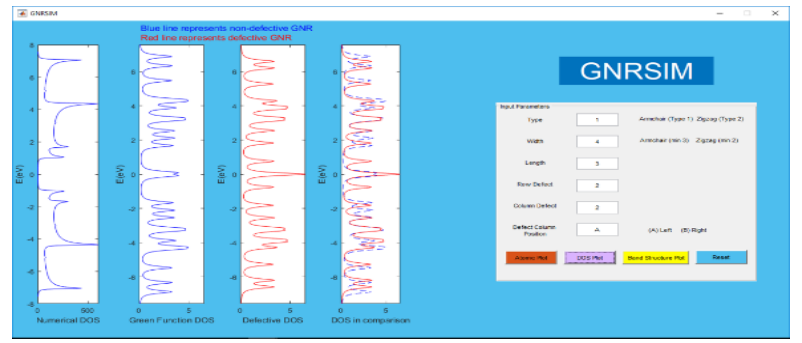

Figure 14. Density of states of 4-AGNR for 3UC length

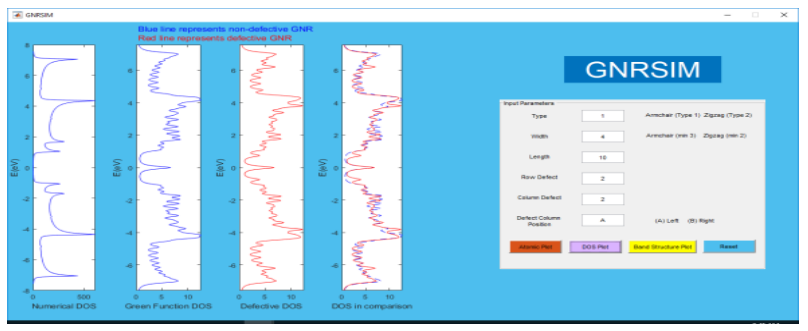

Figure 15. Density of states of 4-AGNR for 10UC length
Table 5. Input Parameters for 4-AGNR with

3UC Length and 4UC Width

\begin{tabular}{|c|c|}
\hline Input Parameters & Value \\
\hline Type & 1 \\
\hline Width & 4 \\
\hline Length & 3 \\
\hline Row Defect & 2 \\
\hline Column Defect & 2 \\
\hline $\begin{array}{ll}\text { Column } & \text { Defect } \\
\text { Position } & \end{array}$ & A \\
\hline
\end{tabular}

Table 6. Input Parameters of 4-AGNR with

\begin{tabular}{|c|c|}
\hline$\frac{100 \mathrm{C} \text { Length a }}{\text { Input Parameters }}$ & Widt \\
\hline Type & 1 \\
\hline Width & 4 \\
\hline Length & 10 \\
\hline Row Defect & 2 \\
\hline Column Defect & 2 \\
\hline $\begin{array}{ll}\text { Column } & \text { Defect } \\
\text { Position } & \end{array}$ & A \\
\hline
\end{tabular}




\section{CONCLUSION}

GNRSIM is a promising educational tool to be utilized by the instructors and researchers towards comprehension and simulation on the electronic property of graphene. It is to be easy to use and provide a wide range of features namely band structure, the density of states based on input parameter. From this research, we identified that the edge type affects GNR electrical properties, which are AGNR and ZGNR. Different AGNR and ZGNR have their own shape of band structure and DOS. ZGNR is always metallic because absent of bandgap while AGNR can be semiconducting or metallic. Besides that, we can compare the electronic properties between pristine and defected GNR when single vacancy defect is introduced inside the atomic structure by using this GNRSIM. The majority of the properties specified holds conceivable leap forward towards a comprehensive computational nanoelectronics in the near future.

\section{ACKNOWLEDGMENTS}

The authors acknowledge financial support from a UTM GUP Research Grant (Vote no. Q.J130000.2523.14H27) that allowed the research to proceed smoothly. Michael L. P. Tan acknowledges the support of the UTM Research Management Centre (RMC) for providing an excellent research environment. Kien Liong Wong would like to express his appreciation for the award of Zamalah PhD Scholarship from the School of Graduate Studies UTM.

\section{REFERENCES}

[1] Sun S, Narayana VK, El-Ghazawi T, Sorger VJ, editors. Chasing Moore's law with CLEAR. 2017 Conference on Lasers and Electro-Optics (CLEO); 2017 14-19 May 2017.

[2] Halfhill TR. The Mythology of Moore's Law: Why Such a Widely Misunderstood 'Law' Is So Captivating to So Many. IEEE Solid-State Circuits Society Newsletter. 2006; 11(3): 21-5.

[3] Neto AHC, Guinea F, Peres NMR, Novoselov KS, Geim AK. The electronic properties of graphene. Rev Mod Phys. 2009; 81.

[4] Novoselov KS, Morozov SV, Mohinddin TMG, Ponomarenko LA, Elias DC, Yang R, Barbolina II, Blake P, Booth TJ, Jiang D, Giesbers J, Hill EW, Geim AK. Electronic properties of graphene. physica status solidi (b). 2007; 244(11): 4106-11.

[5] Moon JS, Gaskill DK. Graphene: Its Fundamentals to Future Applications. IEEE Transactions on Microwave Theory and Techniques. 2011; 59(10): 2702-8.

[6] Cooper DR, D’Anjou B, Ghattamaneni N, Harack B, Hilke M, Horth A, Majlis N, Massicotte M, Vandsburger L, Whiteway E, Yu V. Experimental Review of Graphene. ISRN Condensed Matter Physics. 2012; 2012: 56.

[7] Littlejohn SD. Electrical Properties of Graphite Nanoparticles in Silicone: University of Bath; 2014.

[8] Goh E, Chin HC, Wong KL, Indra ISB, Tan MLP. Modeling and Simulation of the Electronic Properties in Graphene Nanoribbons of Varying Widths and Lengths Using Tight-Binding Hamiltonian. Journal of Nanoelectronics and Optoelectronics. 2018; 13(2): 289-300.

[9] Bhowmick S, Waghmare UV. Anisotropy of the Stone-Wales defect and warping of graphene nanoribbons: A firstprinciples analysis. Physical Review B. 2010; 81(15): 155416.

[10] Tan MLP. Device and Circuit-level Models for Carbon Nanotube and Graphene Nanoribbon Transistors.: University of Cambridge; 2011.

[11] Kliros GS, editor Modeling of carrier density and quantum capacitance in graphene nanoribbon FETs. 2010 International Conference on Microelectronics; 2010 19-22 Dec. 2010.

[12] Schwierz F. Graphene transistors. Nature Nanotechnology. 2010; 5: 487.

[13] Khalid A, Sampe J, Majlis BY, Mohamed MA, Chikuba T, Iwasaki T, Mizuta H, editors. Towards high performance graphene nanoribbon transistors (GNR-FETs). 2015 IEEE Regional Symposium on Micro and Nanoelectronics (RSM); 2015 19-21 Aug. 2015.

[14] Baggott J. The meaning of quantum theory: a guide for students of chemistry and physics. 2000.

[15] D.W. Oxtoby HPG, and L.J. Butler. Principles of modern chemistry. Cengage Learning. 2015.

[16] Datta S, editor The non-equilibrium Green's function (NEGF) formalism: An elementary introduction. Electron Devices Meeting, 2002IEDM'02: International, 2002, pp. 703-706: IEEE.

[17] Datta S. Quantum transport: atom to transistor. Cambridge University Press. 2005.

[18] Datta S. Electronic transport in mesoscopic systems. 1997.

[19] Venugopal R. Modeling quantum transport in nanoscale transistors. Purdue University. 2003.

[20] Tsuneda T. Density Functional Theory in Quantum Chemistry,. Springer Japan. 2014(DOI 10.1007/978-4-43154825-6_2). 